\title{
Palladium-Catalyzed Sonogashira Coupling Reaction of 2-Amino-3-Bromopyridines with Terminal Alkynes
}

\author{
Qing Zhu, Lichun Liao, Guo Cheng, Wen Yang, Yingying Deng, Dingqiao Yang* \\ Key Laboratory of Theoretical Chemistry of Environment, Ministry of Education, School of Chemistry and Environment, South \\ China Normal University, Guangzhou, China \\ Email: *yangdq@scnu.edu.cn
}

How to cite this paper: Zhu, Q., Liao, L.C., Cheng, G., Yang, W., Deng, Y.Y. and Yang, D.Q. (2017) Palladium-Catalyzed Sonogashira Coupling Reaction of 2-Amino-3-Bromopyridines with Terminal Alkynes. Modern Research in Catalysis, 6, 121-133. https://doi.org/10.4236/mrc.2017.63009

Received: June 12, 2017

Accepted: July 10, 2017

Published: July 13, 2017

Copyright (c) 2017 by authors and Scientific Research Publishing Inc. This work is licensed under the Creative Commons Attribution International License (CC BY 4.0).

http://creativecommons.org/licenses/by/4.0/

\section{Open Access}

\begin{abstract}
Palladium-catalyzed the Sonogashira coupling reaction of 3-halogen-2-aminopyridines 1 with terminal alkynes 2 afforded the corresponding 21 target products $3 a-3 \mathbf{u}$ in the presence of palladium catalyst. The structure of target products $3 \mathbf{a}-\mathbf{3} \mathbf{u}$ was confirmed and characterized by ${ }^{1} \mathrm{H} N \mathrm{NM},{ }^{13} \mathrm{C} \mathrm{NMR}$, and HRMS. The influences of different kinds of catalyst loading, bases, substrates and temperature were also investigated. Under the optimized conditions, including $2.5 \mathrm{~mol} \% \mathrm{Pd}\left(\mathrm{CF}_{3} \mathrm{COO}\right)_{2}, 5 \mathrm{~mol} \% \mathrm{PPh}_{3}$ and $5 \mathrm{~mol} \% \mathrm{CuI}$ as additive, 1 $\mathrm{mL} \mathrm{Et}_{3} \mathrm{~N}$, substrate 1 with terminal alkynes 2 for the cross-coupling reactions at $100^{\circ} \mathrm{C}$ for $3 \mathrm{~h}$ in DMF afforded the corresponding products of 2-amino-3alkynylpyridines 3 in moderate to excellent yields (72\% - 96\%). The present methodology has provided an effective synthetic method including operational convenience, high efficiency and wide-application.
\end{abstract}

\section{Keywords}

Palladium Catalyst, 2-Amino-3-Bromopyridines, Terminal Alkynes, Sonogashira Coupling Reaction

\section{Introduction}

Sonogashira reaction is the $\mathrm{sp}-\mathrm{sp}^{2}$ carbon cross-coupling reaction of terminal alkynes with aryl halides or alkenyl halide. This reaction is one of the most effective methods to form new carbon-carbon bonds [1]-[9]. As early as 1975, Heck [10], Cassar [11] and Sonogashira [12] independently discovered this reaction. After nearly 40 years, it has gradually known by people and become an important name reaction. At present, the Sonogashira reaction has been widely used in the synthesis of substituted alkynes and conjugated alkynes [13] [14] [15] [16]. It 
also plays a key role in the synthesis of many natural products [17], pesticides, pharmaceuticals [18] [19] and new materials and nano-molecular devices [20] [21] [22] [23]. Since the discovery of the Sonogashira reactions [24]-[29], the most widely used catalysts have been Pd-type compounds. A lot of cross-coupling reactions catalyzed by $\mathrm{Pd} / \mathrm{Cu}$ co-catalyst are the cross-coupling reactions of terminal alkyne with halogenated benzene, while the reactions of terminal alkynes with halogenated hydrocarbons containing hetero atoms are relatively few investigated. Nitrogen-containing heterocyclic compounds, the substrates of the Sonogashira cross-coupling reactions, of which pyridine and its derivatives are important raw materials in the preparation of pharmaceuticals, pesticides, dyes, surfactants, rubber chemicals, feed additives, food additives, adhesives and composite materials [30]. It also can be used for the preparation of high value-added fine chemicals. The products of 2-amino-3-alkynyl pyridine and its derivatives are important raw materials to synthesize indole and quinoline heterocyclic compounds. The indole containing nitrogen can be thought of as bioisosteres of indole, and indole plays a huge role in the process of life because many medicines contain indole. Azaindole molecules such as 7 -azaindole, an important type of compounds, are widely used in the pharmaceuticals, pesticides, chemicals and food sectors [31] [32]. 7-Azaindole also plays an important role in materials science as metal ligands [33]. Therefore, 2-amino-3-alkynyl pyridine and its derivatives, prepared by cross-coupling reaction have a certain scientific significance and potential application values. One-pot reaction of 2-amino-3-bromopyridine reacts with terminal alkynes to synthesize a series of 2-amino-3-alkynyl pyridine and its derivatives in the presence of palladium catalyst. The few palladium catalytic loading, mild reaction conditions, wide raw material sources and 2-amino-3-bromopyridine and its derivatives with many functional groups make the Sonogashira coupling reaction to successfully explore the optimum reaction conditions, and expand the scope of the substrate 1 . The Sonogashira coupling reaction makes it possible to synthesize, further transform and apply to heterocyclic compounds.

\section{Experimental}

\subsection{General Procedure}

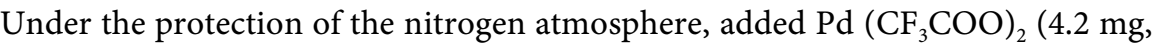
$2.5 \mathrm{~mol} \%), \mathrm{PPh}_{3}$ (6.6 mg, $5.0 \mathrm{~mol} \%$ ), $\mathrm{CuI}(4.8 \mathrm{mg}, 5.0 \mathrm{~mol} \%)$ to $10 \mathrm{~mL}$ roundbottomed flask, then added $2.0 \mathrm{~mL}$ DMF solvent and stirred $30 \mathrm{~min}$. Then added 2-amino-3-bromo-pyridine $1(0.5 \mathrm{mmol})$ with terminal alkynes $2(0.6 \mathrm{mmol})$, in the $100^{\circ} \mathrm{C}$ heating circumfluence $3 \mathrm{~h}$, TLC monitor reaction process. After the reaction, took the reaction mixture into the saturated sodium chloride aqueous solution $(10 \mathrm{~mL})$, extracted with ethyl acetate $(10 \mathrm{~mL} \times 3)$ and collected organic phase, dry with anhydrous magnesium sulfate, filter, vacuum concentrate, wash away residue with petroleum ether and ethyl ester, and the residue is purified by column chromatography to afford the corresponding product 3 . 


\subsection{Spectra Data}

3-Phenylethynyl-2-aminopyridine (3a) [29]. Prepared according to general procedure. A white solid $\left(95.1 \mathrm{mg}, 98 \%\right.$ yield). m.p. $88^{\circ} \mathrm{C}-89^{\circ} \mathrm{C} . \mathrm{R}_{f}=0.40$ on silica gel (ethyl acetate/petroleum ether 1:5, v/v). ${ }^{1} \mathrm{H}$ NMR $\left(500 \mathrm{MHz}, \mathrm{CDCl}_{3}\right) \delta 8.04$ $(\mathrm{dt}, J=10.7,5.3 \mathrm{~Hz}, 1 \mathrm{H}), 7.60(\mathrm{dt}, J=13.9,7.0 \mathrm{~Hz}, 1 \mathrm{H}), 7.56-7.49(\mathrm{~m}, 2 \mathrm{H}), 7.39$ $7.33(\mathrm{~m}, 3 \mathrm{H}), 6.65$ (dd, $J=7.5,5.0 \mathrm{~Hz}, 1 \mathrm{H}), 5.13(\mathrm{~s}, 2 \mathrm{H}) .{ }^{13} \mathrm{C}$ NMR $(125 \mathrm{MHz}$, $\left.\mathrm{CDCl}_{3}\right) \delta 158.8,147.9,134.0,131.5,128.5,128.5,122.7,113.5,103.2,95.5,84.4$. MS (EI) m/z: $[\mathrm{M}+\mathrm{H}]^{+}$calcd for $\mathrm{C}_{13} \mathrm{H}_{11} \mathrm{~N}_{2}, 195.08$; found, 195.15 .

3-(4-Methylphenylethyny)-2-aminopyridine $(3 \mathbf{b})$. Prepared according to general procedure. A light yellow solid $\left(97.8 \mathrm{mg}, 94 \%\right.$ yield). m.p. $94^{\circ} \mathrm{C}-89^{\circ} \mathrm{C} . \mathrm{R}_{f}=$ 0.40 on silica gel (ethyl acetate/petroleum ether 1:5, v/v). ${ }^{1} \mathrm{H}$ NMR $(500 \mathrm{MHz}$, $\left.\mathrm{CDCl}_{3}\right) \delta 8.03(\mathrm{dd}, J=5.0,1.7 \mathrm{~Hz}, 1 \mathrm{H}), 7.58(\mathrm{dd}, J=7.5,1.8 \mathrm{~Hz}, 1 \mathrm{H}), 7.41(\mathrm{~d}, J=$ $8.1 \mathrm{~Hz}, 2 \mathrm{H}), 7.17(\mathrm{~d}, J=7.9 \mathrm{~Hz}, 2 \mathrm{H}), 6.64(\mathrm{dd}, J=7.5,5.0 \mathrm{~Hz}, 1 \mathrm{H}), 5.09(\mathrm{~s}, 2 \mathrm{H})$, 2.37 (s, 3H). ${ }^{13} \mathrm{C}$ NMR $\left(125 \mathrm{MHz}, \mathrm{CDCl}_{3}\right) \delta 158.8,147.8,139.9,138.9,131.4$, 129.3, 119.6, 113.6, 103.4, 95.7, 83.8, 21.5. HRMS (APCI-ion trap) m/z: $[\mathrm{M}+\mathrm{H}]^{+}$ calcd for $\mathrm{C}_{14} \mathrm{H}_{13} \mathrm{~N}_{2}, 209.1000$; found, 209.1001.

3 -(4-Propylphenylethyny)-2-aminopyridine (3c). Prepared according to general procedure. A yellow solid (198.6 mg, $92 \%$ yield). m.p. $92^{\circ} \mathrm{C}-93^{\circ} \mathrm{C} . \mathrm{R}_{f}=0.40$ on silica gel (ethyl acetate/petroleum ether 1:5, v/v). ${ }^{1} \mathrm{H}$ NMR $\left(500 \mathrm{MHz}, \mathrm{CDCl}_{3}\right.$ ) $\delta 8.05-8.00(\mathrm{~m}, 1 \mathrm{H}), 7.58(\mathrm{dd}, J=7.5,1.8 \mathrm{~Hz}, 1 \mathrm{H}), 7.42(\mathrm{t}, J=8.2 \mathrm{~Hz}, 2 \mathrm{H}), 7.16$ $(\mathrm{d}, J=8.1 \mathrm{~Hz}, 2 \mathrm{H}), 6.62(\mathrm{dd}, J=7.5,5.0 \mathrm{~Hz}, 1 \mathrm{H}), 5.18(\mathrm{~s}, 2 \mathrm{H}), 2.61-2.58(\mathrm{~m}$, $2 \mathrm{H}), 1.64(\mathrm{dd}, J=15.0,7.5 \mathrm{~Hz}, 2 \mathrm{H}), 0.94(\mathrm{t}, J=7.3 \mathrm{~Hz}, 3 \mathrm{H}) .{ }^{13} \mathrm{C}$ NMR $(125 \mathrm{MHz}$, $\left.\mathrm{CDCl}_{3}\right) \delta 158.9,147.8,143.7,139.9,131.4,128.7,119.9,113.5,103.4,95.7,83.8$, 38.0, 24.3, 13.8. HRMS (APCI-ion trap) $\mathrm{m} / \mathrm{z}$ : $[\mathrm{M}+\mathrm{H}]^{+}$calcd for $\mathrm{C}_{16} \mathrm{H}_{17} \mathrm{~N}_{2}$, 237.1313; found, 237.1314.

3-Biphenylethynyl-2-aminopyridine (3d). Prepared according to general procedure. Tan oil ( $129.4 \mathrm{mg}, 96 \%$ yield). $\mathrm{R}_{f}=0.42$ on silica gel (ethyl acetate/petroleum ether 1:5, v/v). ${ }^{1} \mathrm{H} \mathrm{NMR}\left(500 \mathrm{MHz}, \mathrm{CDCl}_{3}\right) \delta 8.05(\mathrm{dd}, J=5.0,1.7 \mathrm{~Hz}$, $1 \mathrm{H}), 7.63-7.59(\mathrm{~m}, 7 \mathrm{H}), 7.46(\mathrm{dd}, J=10.5,4.8 \mathrm{~Hz}, 2 \mathrm{H}), 7.37(\mathrm{t}, J=7.4 \mathrm{~Hz}, 1 \mathrm{H})$, $6.67(\mathrm{dd}, J=7.5,5.0 \mathrm{~Hz}, 1 \mathrm{H}), 5.08(\mathrm{~s}, 2 \mathrm{H}) .{ }^{13} \mathrm{C} \mathrm{NMR}\left(125 \mathrm{MHz}, \mathrm{CDCl}_{3}\right) \delta 158.8$, 148.0, 141.4, 140.2, 140.0, 131.9, 128.9, 127.8, 127.1, 127.1, 121.6, 113.7, 103.2, 95.4, 85.1. HRMS (APCI-ion trap) $\mathrm{m} / \mathrm{z}$ : $[\mathrm{M}+\mathrm{H}]^{+}$calcd for $\mathrm{C}_{19} \mathrm{H}_{15} \mathrm{~N}_{2}, 271.1157$; found, 271.1158.

3-Cyclopropylethynyl-2-aminopyridine (3e). Prepared according to general procedure. Yellow oil ( $70.4 \mathrm{mg}, 88 \%$ yield). $\mathrm{R}_{f}=0.35$ on silica gel (ethyl acetate/petroleum ether 1:5, v/v). ${ }^{1} \mathrm{H}$ NMR $\left(500 \mathrm{MHz}, \mathrm{CDCl}_{3}\right) \delta 7.99-7.91(\mathrm{~m}, 1 \mathrm{H})$, 7.45 (dd, $J=7.5,1.8 \mathrm{~Hz}, 1 \mathrm{H}), 6.57$ (dd, $J=7.5,5.1 \mathrm{~Hz}, 1 \mathrm{H}), 4.99$ (s, 2H), 1.49 (tt, $J=8.3,5.0 \mathrm{~Hz}, 1 \mathrm{H}), 0.93-0.88(\mathrm{~m}, 2 \mathrm{H}), 0.81(\mathrm{dt}, J=7.2,4.3 \mathrm{~Hz}, 2 \mathrm{H}) .{ }^{13} \mathrm{C}$ NMR $\left(125 \mathrm{MHz}, \mathrm{CDCl}_{3}\right) \delta 158.9,146.7,139.6,113.1,103.7,99.6,70.6$, 8.6. HRMS (APCI-ion trap) m/z: $[\mathrm{M}+\mathrm{H}]^{+}$calcd for $\mathrm{C}_{10} \mathrm{H}_{11} \mathrm{~N}_{2}, 159.0844$; found, 159.0845.

3-Decynyl-2-aminopyridine (3f). Prepared according to general procedure. Yellow oil (103.5 mg, $90 \%$ yield). $\mathrm{R}_{f}=0.45$ on silica gel (ethyl acetate/petroleum ether 1:5, v/v). ${ }^{1} \mathrm{H}$ NMR (500 MHz, $\left.\mathrm{CDCl}_{3}\right) \delta 7.89(\mathrm{dd}, J=5.0,1.7 \mathrm{~Hz}, 1 \mathrm{H}), 7.38$ (dd, $J=7.5,1.8 \mathrm{~Hz}, 1 \mathrm{H}), 6.49$ (dd, $J=7.5,5.0 \mathrm{~Hz}, 1 \mathrm{H}), 5.07-4.95(\mathrm{~m}, 2 \mathrm{H}), 2.37$ 
(t, $J=7.1 \mathrm{~Hz}, 2 \mathrm{H}), 1.57-1.51(\mathrm{~m}, 2 \mathrm{H}), 1.27-1.17(\mathrm{~m}, 10 \mathrm{H}), 0.80(\mathrm{~d}, J=7.0 \mathrm{~Hz}$, $3 \mathrm{H}) .{ }^{13} \mathrm{C} \mathrm{NMR}\left(125 \mathrm{MHz}, \mathrm{CDCl}_{3}\right) \delta 159.0,147.0,139.7,113.3,104.1,96.9,75.8$, $31.8,29.2,29.1,28.9,28.8,22.7,19.6,14.1$. HRMS (APCI-ion trap) m/z: [M + $\mathrm{H}]^{+}$calcd for $\mathrm{C}_{15} \mathrm{H}_{23} \mathrm{~N}_{2}, 231.1783$; found, 231.1785 .

5-Methyl-3-phenylethynyl-2-aminopyridine ( $3 g$ ). Prepared according to general procedure. A light yellow solid ( $96.7 \mathrm{mg}$, $93 \%$ yield). m.p. $85^{\circ} \mathrm{C}-86^{\circ} \mathrm{C} . \mathrm{R}_{f}=$ 0.40 on silica gel (ethyl acetate/petroleum ether 1:5, v/v). ${ }^{1} \mathrm{H}$ NMR (500 MHz, $\left.\mathrm{CDCl}_{3}\right) \delta 7.80(\mathrm{~d}, J=1.6 \mathrm{~Hz}, 1 \mathrm{H}), 7.43(\mathrm{dt}, J=9.2,2.9 \mathrm{~Hz}, 2 \mathrm{H}), 7.36(\mathrm{dd}, J=9.9$, $1.9 \mathrm{~Hz}, 1 \mathrm{H}), 7.30-7.26(\mathrm{~m}, 3 \mathrm{H}), 4.82(\mathrm{~d}, J=42.9 \mathrm{~Hz}, 2 \mathrm{H}), 2.12(\mathrm{~s}, 3 \mathrm{H}) .{ }^{13} \mathrm{C} \mathrm{NMR}$ $\left(125 \mathrm{MHz}, \mathrm{CDCl}_{3}\right) \delta 156.9,147.9,140.6,131.5,128.5,128.5,122.8,122.6,102.9$, 95.3, 84.6, 17.3. MS (EI) m/z: $[\mathrm{M}+\mathrm{H}]^{+}$calcd for $\mathrm{C}_{14} \mathrm{H}_{13} \mathrm{~N}_{2}, 209.10$; found, 209.23.

5-Methyl-3-(4-methylphenylethynyl)-2-aminopyridine (3h). Prepared according to general procedure. A light yellow solid (123.5 mg, $87 \%$ yield). m.p. $90^{\circ} \mathrm{C}$ $91^{\circ} \mathrm{C} . \mathrm{R}_{f}=0.35$ on silica gel (ethyl acetate/petroleum ether $1: 5, \mathrm{v} / \mathrm{v}$ ). ${ }^{1} \mathrm{H}$ NMR $\left(500 \mathrm{MHz} \mathrm{CDCl}_{3}\right) \delta 7.86(\mathrm{~d}, J=1.7 \mathrm{~Hz}, 1 \mathrm{H}), 7.43(\mathrm{~d}, J=2.0 \mathrm{~Hz}, 1 \mathrm{H}), 7.40(\mathrm{~d}, J=$ $8.1 \mathrm{~Hz}, 2 \mathrm{H}), 7.16(\mathrm{~d}, J=7.9 \mathrm{~Hz}, 2 \mathrm{H}), 4.92(\mathrm{~s}, 2 \mathrm{H}), 2.37$ (s, 3H), $2.18(\mathrm{~s}, 3 \mathrm{H}) .{ }^{13} \mathrm{C}$ NMR $\left(125 \mathrm{MHz}, \mathrm{CDCl}_{3}\right) \delta 156.9,147.7,140.5,138.8,131.4,129.2,122.5,119.7$, 103.1, 95.5, 84.0, 21.5, 17.3. HRMS (APCI-ion trap) $m / z$. $[\mathrm{M}+\mathrm{H}]^{+}$calcd for $\mathrm{C}_{15} \mathrm{H}_{15} \mathrm{~N}_{2}, 223.1157$; found, 223.1158 .

5-Methyl-3-(4-methoxyphenethynyl)-2-aminopyridine (31). Prepared according to general procedure. Tan oil ( $105.9 \mathrm{mg}, 89 \%$ yield). $\mathrm{R}_{f}=0.36$ on silica gel (ethyl acetate/petroleum ether 1:5, v/v). ${ }^{1} \mathrm{H}$ NMR $(500 \mathrm{MHz}, \mathrm{CDCl} 3) \delta 7.85(\mathrm{~d}, J=1.6$ $\mathrm{Hz}, 1 \mathrm{H}$ ), 7.43 (ddd, $J=11.0,6.4,2.3 \mathrm{~Hz}, 3 \mathrm{H}$ ), 6.87 (t, $J=5.7 \mathrm{~Hz}, 2 \mathrm{H}$ ), 5.01 (s, $2 \mathrm{H}), 3.81$ (s, 3H), 2.17 (s, 3H). $\left.{ }^{13} \mathrm{C} \mathrm{NMR} \mathrm{(125} \mathrm{MHz,} \mathrm{CDCl}_{3}\right) \delta$ 159.9, 156.99, $147.5,140.4,133.0,122.4,114.9,114.1,103.3$, 95.3, 83.3, 55.3, 17.3. MS (EI) m/z: $[\mathrm{M}+\mathrm{H}]^{+}$calcd for $\mathrm{C}_{15} \mathrm{H}_{15} \mathrm{~N}_{2} \mathrm{O}, 239.11$; found, 239.18 .

5-Methyl-3-(4-propylphenylethynyl)-2-aminopyridine (3j). Prepared according to general procedure. A yellow solid (110.9 mg, $89 \%$ yield). m.p. $88^{\circ} \mathrm{C}-89^{\circ} \mathrm{C}$. $\mathrm{R}_{f}=0.40$ on silica gel (ethyl acetate/petroleum ether $\left.1: 5, \mathrm{v} / \mathrm{v}\right) .{ }^{1} \mathrm{H}$ NMR $(500$ $\left.\mathrm{MHz}, \mathrm{CDCl}_{3}\right) \delta 7.79(\mathrm{~d}, J=1.6 \mathrm{~Hz}, 1 \mathrm{H}), 7.35(\mathrm{dd}, J=8.1,1.7 \mathrm{~Hz}, 3 \mathrm{H}), 7.09(\mathrm{~d}, J=$ $8.2 \mathrm{~Hz}, 2 \mathrm{H}), 4.85(\mathrm{~s}, 2 \mathrm{H}), 2.54-2.50(\mathrm{~m}, 2 \mathrm{H}), 2.11(\mathrm{~s}, 3 \mathrm{H}), 1.57(\mathrm{dd}, J=15.0,7.5$ $\mathrm{Hz}, 2 \mathrm{H}), 0.87(\mathrm{t}, J=7.3 \mathrm{~Hz}, 3 \mathrm{H}) .{ }^{13} \mathrm{C} \mathrm{NMR}\left(125 \mathrm{MHz}, \mathrm{CDCl}_{3}\right) \delta 156.9,147.7$, $143.6,140.5,131.4,128.6,122.5,120.0,103.1,95.5,84.0,38.0,24.3,17.3,13.8$. HRMS (APCI-ion trap) $\mathrm{m} / z:[\mathrm{M}+\mathrm{H}]^{+}$calcd for $\mathrm{C}_{17} \mathrm{H}_{19} \mathrm{~N}_{2}, 251.1470$; found, 251.1471 .

5-Methyl-3-biphenyl ethynyl-2-aminopyridine (3k). Prepared according to general procedure. Tan oil (123.4 mg, $90 \%$ yield). $\mathrm{R}_{f}=0.41$ on silica gel (ethyl acetate/petroleum ether 1:5, v/v). ${ }^{1} \mathrm{H}$ NMR $(500 \mathrm{MHz}, \mathrm{CDCl} 3) \delta 7.89(\mathrm{~d}, J=1.3$ $\mathrm{Hz}, 1 \mathrm{H}), 7.63-7.58(\mathrm{~m}, 6 \mathrm{H}), 7.46(\mathrm{dd}, J=9.5,5.3 \mathrm{~Hz}, 3 \mathrm{H}), 7.26(\mathrm{~s}, 1 \mathrm{H}), 4.95$ (s, 2H), 2.21 (s, 3H). ${ }^{13} \mathrm{C} \mathrm{NMR}\left(125 \mathrm{MHz} \mathrm{CDCl}_{3}\right) \delta 156,147.9,141.4,140.7,140.2$ 131.9, 128.9, 127.8, 127.1, 127.1, 122.6, 121.7, 95.3, 85.3, 17.3. HRMS (APCI-ion trap) $\mathrm{m} / \mathrm{z}:[\mathrm{M}+\mathrm{H}]^{+}$calcd for $\mathrm{C}_{20} \mathrm{H}_{17} \mathrm{~N}_{2}, 285.1313$; found, 285.1314.

4-Methyl-3-( $\alpha$-thienylethynyI)-2-aminopyridine (3I). Prepared according to general procedure. Yellow oil $\left(95.2 \mathrm{mg}, 89 \%\right.$ yield). $\mathrm{R}_{f}=0.40$ on silica gel (ethyl 
acetate/petroleum ether 1:5, v/v). ${ }^{1} \mathrm{H}$ NMR (500 MHz, $\left.\mathrm{CDCl}_{3}\right) \delta 7.96(\mathrm{t}, J=6.0$ $\mathrm{Hz}, 1 \mathrm{H}), 7.47-7.42(\mathrm{~m}, 2 \mathrm{H}), 6.91-6.86(\mathrm{~m}, 2 \mathrm{H}), 5.14(\mathrm{~s}, 2 \mathrm{H}), 3.83(\mathrm{~s}, 3 \mathrm{H}) .{ }^{13} \mathrm{C}$ NMR $\left(125 \mathrm{MHz}, \mathrm{CDCl}_{3}\right) \delta 160.2,157.1,145.9,138.8,133.1,120.1,114.2,104.8$, 96.6, 82.0, 55.4. HRMS (APCI-ion trap) $\mathrm{m} / \mathrm{z}$ : $[\mathrm{M}+\mathrm{H}]^{+}$calcd for $\mathrm{C}_{12} \mathrm{H}_{11} \mathrm{~N}_{2} \mathrm{~S}$, 215.0565; found, 215.0566 .

5-Methyl-3-cyclopropylethynyl-2-aminopyridine $(\mathbf{3 m})$. Prepared according to general procedure. Yellow oil $\left(76.5 \mathrm{mg}, 89 \%\right.$ yield). $\mathrm{R}_{f}=0.40$ on silica gel (ethyl acetate/petroleum ether 1:5, v/v). ${ }^{1} \mathrm{H} \mathrm{NMR}\left(500 \mathrm{MHz}, \mathrm{CDCl}_{3}\right) \delta 7.79(\mathrm{~d}, J=1.5$ $\mathrm{Hz}, 1 \mathrm{H}), 7.29(\mathrm{~d}, J=2.0 \mathrm{~Hz}, 1 \mathrm{H}), 4.80(\mathrm{~s}, 2 \mathrm{H}), 2.14(\mathrm{~s}, 3 \mathrm{H}), 1.48(\mathrm{tt}, J=8.3,5.0$ $\mathrm{Hz}, 1 \mathrm{H}), 0.90(\mathrm{dt}, J=8.1,3.1 \mathrm{~Hz}, 2 \mathrm{H}), 0.80(\mathrm{dt}, J=7.2,4.3 \mathrm{~Hz}, 2 \mathrm{H}) .{ }^{13} \mathrm{C} \mathrm{NMR}$ $\left(125 \mathrm{MHz}, \mathrm{CDCl}_{3}\right) \delta 157.2,146.8,140.6,122.4,103.7,99.7,71.0,17.2,8.9$. HRMS (APCI-ion trap) m/z: $[\mathrm{M}+\mathrm{H}]^{+}$calcd for $\mathrm{C}_{11} \mathrm{H}_{13} \mathrm{~N}_{2}, 173.1000$; found, 173.1002 .

5-Methyl-3-decynyl-2-aminopyridine (3n). Prepared according to general procedure. Yellow oil ( $102.5 \mathrm{mg}, 84 \%$ yield). $\mathrm{R}_{f}=0.35$ on silica gel (ethyl acetate/petroleum ether 1:5, v/v). ${ }^{1} \mathrm{H} \mathrm{NMR}\left(500 \mathrm{MHz}, \mathrm{CDCl}_{3}\right) \delta 7.80(\mathrm{~d}, J=1.6 \mathrm{~Hz}$, $1 \mathrm{H}), 7.31(\mathrm{~d}, J=2.1 \mathrm{~Hz}, 1 \mathrm{H}), 4.81(\mathrm{~s}, 2 \mathrm{H}), 2.44(\mathrm{t}, J=7.1 \mathrm{~Hz}, 2 \mathrm{H}), 2.15(\mathrm{~s}, 3 \mathrm{H})$, $1.37-1.21(\mathrm{~m}, 12 \mathrm{H}), 0.89(\mathrm{t}, J=6.7 \mathrm{~Hz}, 3 \mathrm{H}) .{ }^{13} \mathrm{C} \mathrm{NMR}\left(125 \mathrm{MHz}, \mathrm{CDCl}_{3}\right) \delta$ 157.1, 146.8, 146.8, 140.5, 122.4, 96.7, 75.9, 31.8, 29.3, 29.1, 28.9, 28.8, 22.7, 19.6, 17.2, 14.1. HRMS (APCI-ion trap) $\mathrm{m} / \mathrm{z}:[\mathrm{M}+\mathrm{H}]^{+}$calcd for $\mathrm{C}_{16} \mathrm{H}_{25} \mathrm{~N}_{2}, 245.1939$; found, 245.1940 .

3-Phenylethynyl-2-amino-5-chloropyridine (3o). Prepared according to general procedure. A white solid (101.4 mg, 89\% yield). m.p. $84^{\circ} \mathrm{C}-85^{\circ} \mathrm{C} . \mathrm{R}_{f}=0.40$ on silica gel (ethyl acetate/petroleum ether 1:5, v/v). ${ }^{1} \mathrm{H}$ NMR (500 $\mathrm{MHz}, \mathrm{CDCl}_{3}$ ) $\delta 7.98(\mathrm{~d}, J=2.5 \mathrm{~Hz}, 1 \mathrm{H}), 7.56(\mathrm{~d}, J=2.5 \mathrm{~Hz}, 1 \mathrm{H}), 7.52-7.49(\mathrm{~m}, 2 \mathrm{H}), 7.37(\mathrm{dt}, J=$ $4.5,2.3 \mathrm{~Hz}, 3 \mathrm{H}), 5.18(\mathrm{~s}, 2 \mathrm{H}) .{ }^{13} \mathrm{C} \mathrm{NMR}\left(125 \mathrm{MHz}, \mathrm{CDCl}_{3}\right) \delta 157.2,146.4,139.1$, 131.6, 129.0, 128.6, 122.2, 120.1, 104.3, 96.5, 83.2. HRMS (APCI-ion trap) m/z: $[\mathrm{M}+\mathrm{H}]^{+}$calcd for $\mathrm{C}_{13} \mathrm{H}_{10} \mathrm{ClN}_{2}, 229.0454$; found, 229.0455 .

3-(4-Propylphenylethynyl)-2-amino-5-chloropyridine ( $3 p$ ). Prepared according to general procedure. A Yellow solid (110.9 mg, 85\% yield). m.p. $83^{\circ} \mathrm{C}$ $84^{\circ} \mathrm{C} . \mathrm{R}_{f}=0.40$ on silica gel (ethyl acetate/petroleum ether $1: 5, \mathrm{v} / \mathrm{v}$ ). ${ }^{1} \mathrm{H} \mathrm{NMR}$ $\left(500 \mathrm{MHz}, \mathrm{CDCl}_{3}\right) \delta 7.97(\mathrm{~d}, J=1.9 \mathrm{~Hz}, 1 \mathrm{H}), 7.58-7.54(\mathrm{~m}, 1 \mathrm{H}), 7.42(\mathrm{~d}, J=8.2$ $\mathrm{Hz}, 2 \mathrm{H}), 7.18(\mathrm{~d}, J=8.2 \mathrm{~Hz}, 2 \mathrm{H}), 5.11(\mathrm{~s}, 2 \mathrm{H}), 2.62-2.59(\mathrm{~m}, 2 \mathrm{H}), 1.64(\mathrm{t}, J=6.0$ $\mathrm{Hz}, 2 \mathrm{H}), 0.96-0.93(\mathrm{~m}, 3 \mathrm{H}) .{ }^{13} \mathrm{C} \mathrm{NMR}\left(125 \mathrm{MHz}, \mathrm{CDCl}_{3}\right) \delta 157.1,146.1,144.1$, 138.9, 131.6, 131.6, 128.9 128.6, 128.4, 119.3, 96.8, 82.6, 38.0, 24.3, 13.8. HRMS (APCI-ion trap) m/z: $[\mathrm{M}+\mathrm{H}]^{+}$calcd for $\mathrm{C}_{16} \mathrm{H}_{16} \mathrm{ClN}_{2}, 271.0924$; found, 271.0925 .

3-Decynyl-2-amino-5-chloropyridine (3q). Prepared according to general procedure. Colorless oil ( $118.8 \mathrm{mg}, 90 \%$ yield). $\mathrm{R}_{f}=0.45$ on silica gel (ethyl acetate/petroleum ether 1:5, v/v). ${ }^{1} \mathrm{H}$ NMR $\left(500 \mathrm{MHz}, \mathrm{CDCl}_{3}\right) \delta 7.91(\mathrm{~d}, J=2.4 \mathrm{~Hz}$, $1 \mathrm{H}), 7.43(\mathrm{~d}, J=2.5 \mathrm{~Hz}, 1 \mathrm{H}), 5.13(\mathrm{~s}, 2 \mathrm{H}), 2.44(\mathrm{t}, J=7.1 \mathrm{~Hz}, 2 \mathrm{H}), 1.43(\mathrm{dd}, J=$ 10.1, $4.7 \mathrm{~Hz}, 2 \mathrm{H}), 1.35-1.24(\mathrm{~m}, 10 \mathrm{H}), 0.89(\mathrm{t}, J=7.0 \mathrm{~Hz}, 3 \mathrm{H}) .{ }^{13} \mathrm{C} \mathrm{NMR}(125$ $\left.\mathrm{MHz}, \mathrm{CDCl}_{3}\right) \delta 157.5,145.3,138.9,119.8,105.3,98.2,74.9,31.8,29.3$ 29.1, 28.9, 28.6, 22.7, 19.6, 14.1. HRMS (APCI-ion trap) $\mathrm{m} / \mathrm{z}$ : $[\mathrm{M}+\mathrm{H}]^{+}$calcd for $\mathrm{C}_{15} \mathrm{H}_{22} \mathrm{ClN}_{2}$, 265.1393; found, 265.1395.

4-Methyl-3-(a-thienylethynyl)-2-amino-5-bromopyridine (3r). Prepared ac- 
cording to general procedure. Colorless oil (113.8 mg, $78 \%$ yield). $\mathrm{R}_{f}=0.40$ on silica gel (ethyl acetate/petroleum ether 1:5, v/v). ${ }^{1} \mathrm{H}$ NMR $\left(500 \mathrm{MHz}, \mathrm{CDCl}_{3}\right) \delta$ $7.88(\mathrm{~d}, J=1.7 \mathrm{~Hz}, 1 \mathrm{H}), 7.43(\mathrm{t}, J=4.6 \mathrm{~Hz}, 1 \mathrm{H}), 7.33-7.29(\mathrm{~m}, 1 \mathrm{H}), 7.28-7.26$ $(\mathrm{m}, 1 \mathrm{H}), 4.96(\mathrm{~d}, J=42.1 \mathrm{~Hz}, 2 \mathrm{H}), 2.19(\mathrm{~s}, 3 \mathrm{H}) .{ }^{13} \mathrm{C} \mathrm{NMR}\left(125 \mathrm{MHz}, \mathrm{CDCl}_{3}\right) \delta$ $156.8,148.2,140.6,132.1,127.7,127.2,122.8,122.6,102.5,88.3,88.3,17.3$. HRMS (APCI-ion trap) m/z: $[\mathrm{M}+\mathrm{H}]^{+}$calcd for $\mathrm{C}_{12} \mathrm{H}_{10} \mathrm{BrN}_{2} \mathrm{~S}, 292.9670$; found, 292.9671.

4-Methyl-3-cyclopropylethynyl-2-amino-5-bromopyridine (3s). Prepared according to general procedure. Light brown oil ( $90.3 \mathrm{mg}, 72 \%$ yield). $\mathrm{R}_{f}=0.43$ on silica gel (ethyl acetate/petroleum ether 1:5, v/v). ${ }^{1} \mathrm{H}$ NMR $\left(500 \mathrm{MHz}, \mathrm{CDCl}_{3}\right) \delta$ $7.73(\mathrm{dd}, J=12.0,1.7 \mathrm{~Hz}, 1 \mathrm{H}), 4.75(\mathrm{~d}, J=42.5 \mathrm{~Hz}, 2 \mathrm{H}), 2.06(\mathrm{~s}, 3 \mathrm{H}), 1.41(\mathrm{tt}, J=$ 8.3, $5.0 \mathrm{~Hz}, 1 \mathrm{H}), 0.87-0.77(\mathrm{~m}, 2 \mathrm{H}), 0.70(\mathrm{ddt}, J=12.4,6.3,4.0 \mathrm{~Hz}, 2 \mathrm{H}) .{ }^{13} \mathrm{C}$ NMR (125 MHz, $\left.\mathrm{CDCl}_{3}\right) \delta 157.0,146.5,140.3,122.0,103.3,99.3,70.7,16.9$, 8.6. MS (EI) m/z: $[\mathrm{M}+\mathrm{H}]^{+}$calcd for $\mathrm{C}_{11} \mathrm{H}_{12} \mathrm{BrN}_{2}, 251.0106$; found, 251.0107.

5-Trifluoromethyl-3-phenylethynyl-2-aminopyridine (3t). Prepared according to general procedure. A light yellow solid (119.2 mg, $91 \%$ yield). m.p. $79^{\circ} \mathrm{C}$ $79^{\circ} \mathrm{C} . \mathrm{R}_{f}=0.40$ on silica gel (ethyl acetate/petroleum ether $1: 5$, v/v). ${ }^{1} \mathrm{H}$ NMR $\left(500 \mathrm{MHz}, \mathrm{CDCl}_{3}\right) \delta 8.27(\mathrm{~s}, 1 \mathrm{H}), 7.80(\mathrm{~d}, J=2.0 \mathrm{~Hz}, 1 \mathrm{H}), 7.53$ (dd, $J=6.5,3.1$ $\mathrm{Hz}, 2 \mathrm{H}), 7.42-7.34(\mathrm{~m}, 3 \mathrm{H}), 5.66(\mathrm{~s}, 2 \mathrm{H}) .{ }^{13} \mathrm{C} \mathrm{NMR}\left(126 \mathrm{MHz}, \mathrm{CDCl}_{3}\right) \delta 160.5$, $145.3,145.3,136.9,136.9,131.6,129.2,128.6,122.0,102.9,96.6,82.9 . \mathrm{MS}$ (EI) $\mathrm{m} / \mathrm{z}:[\mathrm{M}+\mathrm{H}]^{+}$calcd for $\mathrm{C}_{14} \mathrm{H}_{9} \mathrm{~F}_{3} \mathrm{~N}_{2}, 263.07$; found, 263.08 .

5-Carboxy-3-phenylethynyl-2-aminopyridine (3u). Prepared according to general procedure. Tan oil ( $107.1 \mathrm{mg}, 90 \%$ yield). $\mathrm{R}_{f}=0.40$ on silica gel (ethyl acetate/petroleum ether 1:5, v/v). ${ }^{1} \mathrm{H}$ NMR $(500 \mathrm{MHz}, \mathrm{CDCl} 3) \delta 8.07(\mathrm{~d}, J=2.3$ $\mathrm{Hz}, 1 \mathrm{H}), 7.69$ (d, $J=2.3 \mathrm{~Hz}, 1 \mathrm{H}), 7.51(\mathrm{dd}, J=6.6,3.0 \mathrm{~Hz}, 2 \mathrm{H}), 7.37$ (dd, $J=5.0$, $1.7 \mathrm{~Hz}, 3 \mathrm{H}), 5.17$ (s, 2H). ${ }^{13} \mathrm{C} \mathrm{NMR}\left(126 \mathrm{MHz}, \mathrm{CDCl}_{3}\right) \delta 157.4,148.5,141.6$, $131.5,131.5,129.0,128.6,122.2,107.1,104.9,96.6,83.1$. HRMS (APCI-ion trap) $\mathrm{m} / \mathrm{z}:[\mathrm{M}+\mathrm{H}]^{+}$calcd for $\mathrm{C}_{14} \mathrm{H}_{9} \mathrm{~F}_{3} \mathrm{~N}_{2}, 239.0742$; found, 239.0741 .

\section{Results and Discussion}

To get the best reaction conditions, we chose the cross-coupling of 2-amino-3bromopyridine $(0.5 \mathrm{mmol})$ (1a) with phenylacetylene $(0.6 \mathrm{mmol})(2 \mathrm{a})$ as the model reaction. We optimized the reaction conditions by using different bases and solvents, as well as changing the reaction temperature. The results are summered in Table 1. First, we used $\mathrm{Pd}$ complexes combined with $\mathrm{Pd}(\mathrm{OAc})_{2}(2.5$ mol\%) and $\mathrm{PPh}_{3}(5 \mathrm{~mol} \%)$ as catalysts, $\mathrm{CuI}$ as a co-catalyst, $\mathrm{Et}_{3} \mathrm{~N}$ as base, dimethyl formamide (DMF) as a solvent for $3 \mathrm{~h}$ under $100^{\circ} \mathrm{C}$, the product $3 \mathrm{a}$ was obtained in the yield of $79 \%$ (Table 1, entry 1). Then, we began to optimize the Pdtype catalysts, such as $\mathrm{Pd}(\mathrm{OAc})_{2} / \mathrm{PPh}_{3}, \mathrm{PdCl}_{2}\left(\mathrm{PPh}_{3}\right)_{2} / \mathrm{PPh}_{3}$, and $\mathrm{Pd}\left(\mathrm{CF}_{3} \mathrm{COO}\right)_{2} /$ $\mathrm{PPh}_{3}$. When chose $\mathrm{Pd}\left(\mathrm{CF}_{3} \mathrm{COO}\right)_{2} / \mathrm{PPh}_{3}$ as a catalyst, afforded the corresponding product $3 \mathrm{a}$ in the yield of $96 \%$ (Table 1 , entries 1 - 3). The amount loading of catalyst is also optimized. We chose $\mathrm{Pd}\left(\mathrm{CF}_{3} \mathrm{COO}\right)_{2} / \mathrm{PPh}_{3}$ as the catalyst, we found that the yield increased with the increase the amount of catalyst loading. When the amount of catalyst loading increased from $0.25 \mathrm{~mol} \%$ to $0.50 \mathrm{~mol} \%$, 
Table 1. Optimization of reaction conditions ${ }^{\mathrm{a}}$.

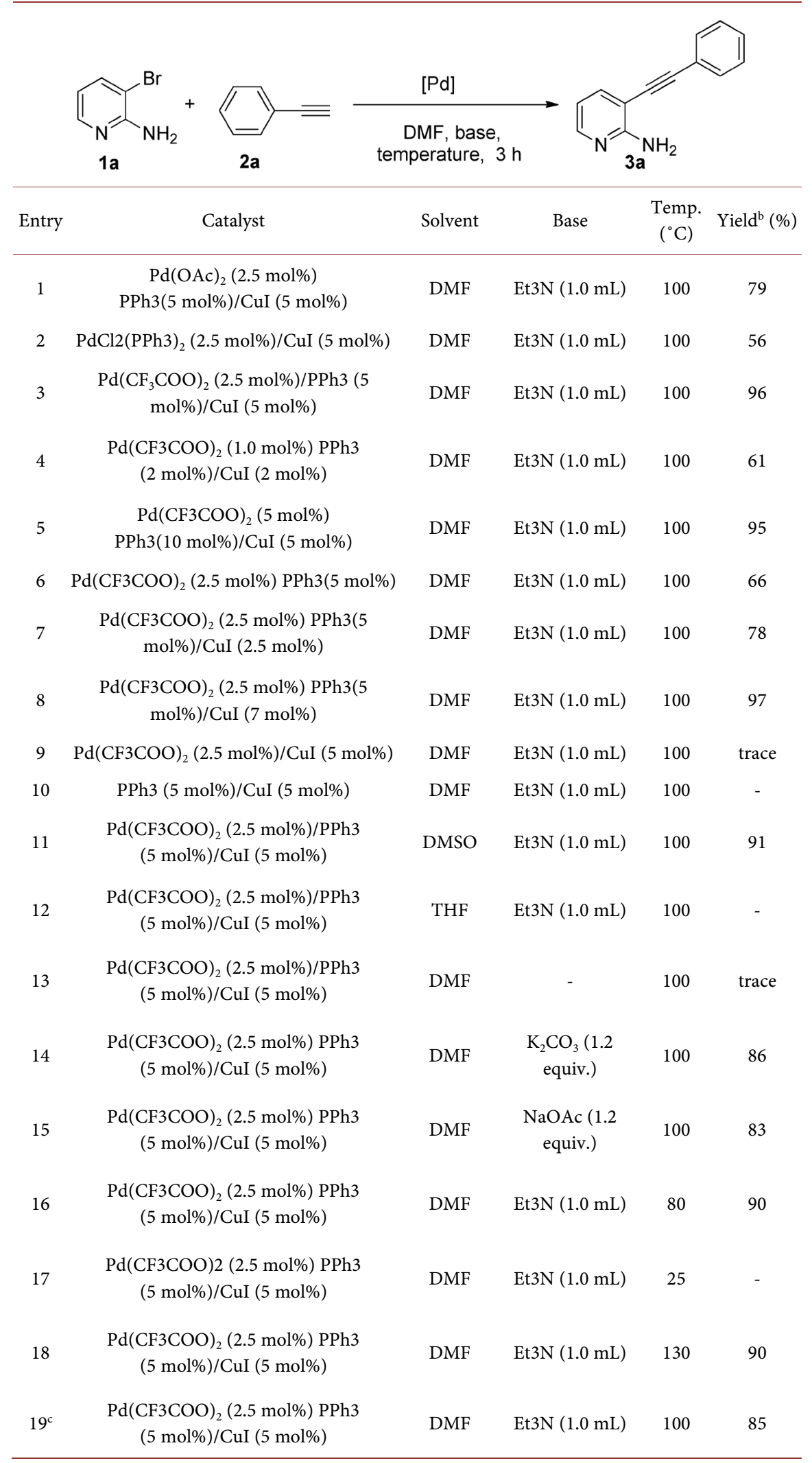

${ }^{\mathrm{a}}$ Reaction conditions: Pd Catalyst, $\mathrm{CuI}$ and PPh3 in DMF $(2 \mathrm{~mL})$ were stirred for 30 min under $\mathrm{N} 2 \mathrm{~atm}$. Then, $1 \mathbf{a}(0.5 \mathrm{mmol})$ with $\mathbf{2 a}$ ( 1.2 equiv.) were added, and the reaction mixture was stirred for $3 \mathrm{~h}$. ${ }^{\mathrm{b}}$ Isolated yields after column chromatography. 'Without N2 protection. 
the yield was not changed. If we kept the same amount of palladium catalyst, changed the loading amount of the cuprous iodide, reaction yield has big changes. Without the cuprous iodide as additive, the yield is $66 \%$ (Table 1, entry 6); Continue to optimize the cuprous salt, we found that with the increment loading amount of cuprous iodide, the yield was increased, while it increased to $7 \mathrm{~mol} \%$, the yield was increased to be $97 \%$ (Table 1, entries 7 - 8). If no palladium only cuprous iodide as additive in the reaction, the reaction cannot occur (Table 1, entry 9). If only have $\mathrm{Pd}\left(\mathrm{CF}_{3} \mathrm{COO}\right)_{2}$ without triphenylphosphine, only a small amount of product $3 \mathbf{a}$ by TLC testing (Table 1, entry 10). Next, the solvent was optimized, the reaction cannot be carried out in tetrahydrofuran (THF). It has a high yield when we chose $N, N$-dimethyl formamide (DMF) or dimethyl sulfoxide (DMSO) as solvents, while has a higher yield to use DMF as a solvent (Table 1, entries 3, $11-12$ ). We found that the bases was also affected the cross-coupling reaction. It only produced trace amounts of products without base (Table 1, entry 13); Therefore, we added potassium carbonate or sodium acetate as the bases for the cross-coupling reaction (Table 1, entries 14 - 15). Temperature also influences the Sonogashira coupling reactions. At ambient temperature conditions, coupling reactions cannot occur, as temperatures rise, the yields have greatly improved; but when the temperature continues to rise, there is a slight fall in yield (Table 1, entries 16 - 18). It may be due to rising temperatures make alkynes couple itself, producing by-products. So the best coupling reaction temperature is $100^{\circ} \mathrm{C}$. In the absence of nitrogen atmosphere protection, the yield is $85 \%$, which indicates that the reaction can also occur in the air (Table 1, entry 19). Therefore, the optimal reaction conditions are 2.5 $\mathrm{mol} \% \mathrm{Pd}\left(\mathrm{CF}_{3} \mathrm{COO}\right)_{2}$ as catalyst, $5 \mathrm{~mol} \% \mathrm{PPh}_{3}$ as ligand, $5 \mathrm{~mol} \% \mathrm{CuI}$ as additive, $1 \mathrm{~mL} \mathrm{Et}_{3} \mathrm{~N}$ as base, DMF as solvent, reaction time $3 \mathrm{~h}$, and temperature $100^{\circ} \mathrm{C}$ for the Sonogashira cross-coupling reaction.

After the optimal reaction conditions, we began to expand the different substrates 1, these substrates react with all kinds of terminal alkynes 2 afforded the corresponding products 3 in good yields (up to 96\%) in Table 2 .

First of all, we took 2-amino-3-bromopyridine 1a as the substrate and found that when it reacts with various aryl acetylene compounds, the yields are more than 90\% (Table 2, entries 1 - 4); When 2-amino-3-bromopyridine 1a reacts with cyclopropyl acetylene, the yield is $88 \%$ (Entry 5); When 1-decyne reacts with 2-amino-3-bromopyridine $1 \mathrm{a}$, the yield of which is slightly lower, just $85 \%$ (Table 2, entry 6). When we took pyridine ring with electronic pushing substituents 2 -amino-3-bromo-5-methyl pyridine $1 \mathrm{~b}$ as a substrate, $1 \mathrm{~b}$ reacted with various terminal alkynes 2, the yields are up to 93\% (Table 2, entries 7 - 14). When we took pyridine ring with electronic withdrawing substituents $1 \mathrm{c}, 1 \mathrm{e}$, and $1 \mathrm{f}$ as substrates, no matter react with terminal aryl acetylene, or with the terminal alkyl acetylene, the yields are up to 91\% (Table 2, entries 15 - 17 and 21 22); But with 2,5-dibromo-4-methyl-pyridin-3-yl amine $1 \mathrm{~d}$ as a substrate, the yield of the product decreased (Table 2, entries 18 - 19); And with 2-bromo-5-nitro-pyridin-3-yl amine $1 \mathrm{~g}$ as a substrate, the coupling reaction is not 
Table 2. Coupling scope of palladium-catalyzed 2-amino-3-bromopyridines with various terminal alkynes ${ }^{\mathrm{a}}$.

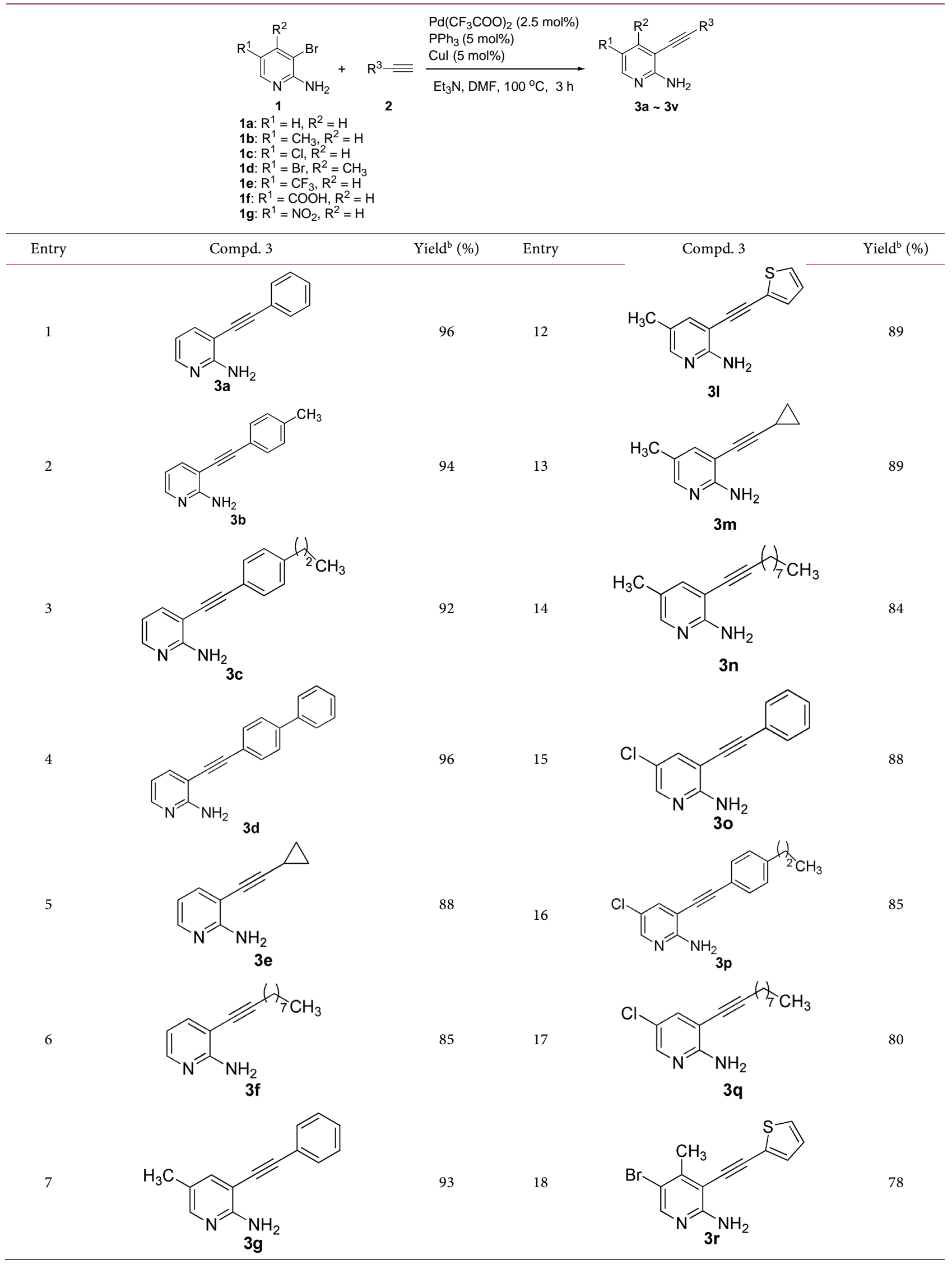




\section{Continued}<smiles>Cc1ccc(C#Cc2cc(C)cnc2N)cc1</smiles><smiles>COc1ccc(C#Cc2cc(C)cnc2N)cc1</smiles>

10<smiles>C[C]c1ccc(C#Cc2cc(C)cnc2N)cc1</smiles>

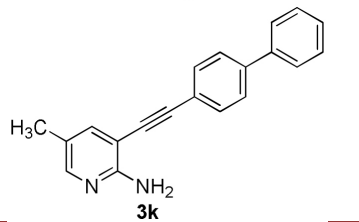

87

19<smiles>Cc1c(Br)cnc(N)c1C#CC1CC1</smiles>

89

20

89

21<smiles>Nc1ncc(C(=O)O)cc1C#Cc1ccccc1</smiles>

22<smiles>Nc1ncc(C(F)(F)F)cc1C#Cc1ccccc1</smiles>

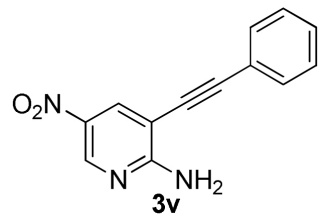

a Reaction conditions: $\mathrm{Pd}\left(\mathrm{CF}_{3} \mathrm{COO}\right)_{2}(2.5 \mathrm{~mol} \%), \mathrm{PPh}_{3}(5 \mathrm{~mol} \%)$ and $\mathrm{CuI}(5 \mathrm{~mol} \%)$ in DMF $(2 \mathrm{~mL})$ was stirred for $30 \mathrm{~min}$ under $\mathrm{N}_{2}$ atm. Then, $1(0.5 \mathrm{mmol})$ with 2 ( 1.2 equiv) were added, and the reaction mixture was stirred at $100^{\circ} \mathrm{C}$ for $3 \mathrm{~h}$. ${ }^{b}$ Isolated yields after column chromatography.

able to occur (Table 2, entry 22).

\section{Conclusion}

In summary, we have developed a simple and efficient method for preparing functionalized the 2-amino-3-ynylpyridine and its derivatives 3 via 2 -amino-3bromopyridines 1 with a serious of terminal alkynes 2 catalyzed by palladium catalyst. A wide range of terminal alkynes were suitable to give the corresponding 2-amino-3-ynylpyridine and its derivative products 3 with high yields. On the other hand, the 2-amino-3-ynylpyridines 3 are the essential raw materials for the synthesis of the heterocycle compounds, such as azaindole, pyridines and quinolines. Further development of coupling reactions is underway in our laboratories and will be reported in due course.

\section{Acknowledgements}

We gratefully thank the National Natural Science Foundation of China (21172081, 21372090), the Natural Science Foundation of Guangdong Province (S2013020013091), the City of Guangzhou Science and Technology Plan Projects (201510010054), and Graduate Student Research and Innovation Foundation of South China Normal University (2015lkxm32) for financial support.

\section{Competing Interests}

Authors have declared that no competing interests exist. 


\section{References}

[1] Manashi, S., Manoj, M., Shivanee Borpatra, G. and Utpal, B. (2017) Gallic AcidDerived Palladium(0) Nanoparticles as in Situ-Formed Catalyst for Sonogashira Cross-Coupling Reaction in Ethanol under Open Air. Catalysis Communications, 90, 31-34. https://doi.org/10.1016/j.catcom.2016.10.034

[2] Christopher, W.D., Ming, G. and Navarro, C.O. (2014) Sonogashira Couplings Catalyzed by Collaborative (N-Heterocyclic Carbene)-Copper and -Palladium Complexes. Organic Letters, 16, 3724-3727. https://doi.org/10.1021/ol501540w

[3] Fujino, T., Hinoue, T., Usukiand, Y. and Satoh, T. (2016) Synthesis of Difluorinated Enynes through Sonogashira-Type Coupling. Organic Letters, 18, 5688-5691. https://doi.org/10.1021/acs.orglett.6b02919

[4] Chinchilla, R. and Nájera, C. (2007) The Sonogashira Reaction: A Booming Methodology in Synthetic Organic Chemistry. Chemical Reviews, 107, 874-922. https://doi.org/10.1021/cr050992x

[5] Doucet, H. and Hierso, J.C. (2007) Palladium-Based Catalytic Systems for the Synthesis of Conjugated Enynes by Sonogashira Reactions and Related Alkynylations. Angewandte Chemie, International Edition, 46, 834-871. https://doi.org/10.1002/anie.200602761

[6] Beccalli, E.M., Broggini, G., Martinelli, M. and Sottocornola, S. (2007) C-C, C-O, C-N Bond Formation on $\mathrm{sp}^{2}$ Carbon by Palladium (II)-Catalyzed Reactions Involving Oxidant Agents. Chemical Reviews, 107, 5318-5365. https://doi.org/10.1021/cr068006f

[7] Alberico, D., Scott, M.E. and Lautens, M. (2007) Aryl-Aryl Bond Formation by Transition-Metal-Catalyzed Direct Arylation. Chemical Reviews, 107, 174-238. https://doi.org/10.1021/cr0509760

[8] Negishi, E.-I. and Anastasia, L. (2003) Palladium-Catalyzed Alkynylation. Chemical Reviews, 103, 1979-2017. https://doi.org/10.1021/cr020377i

[9] Takahashi, S., Kuroyama, Y., Sonogashira, K. and Hagihara, N. (1980) The MetalAmmonia Reduction of 1-Naphthoic Acid. Synthesis, 10, 627-632. https://doi.org/10.1055/s-1980-29145

[10] Diek, H.A. and Heck, F.R. (1975) Palladium Catalyzed Synthesis of Aryl, Heterocyclic, and Vinylic Acetylene Derivatives. Journal of Organometallic Chemistry, 93, 259-263. https://doi.org/10.1016/S0022-328X(00)94049-X

[11] Cassar, L. (1975) Synthesis of Aryl- and Vinyl-Substituted Acetylene Derivatives by the Use of Nickel and Palladium Complexes. Journal of Organometallic Chemistry, 93, 253-257.

[12] Sonogashira, K., Tohda, Y. and Hagihara, N. (1975) A Convenient Synthesis of Acetylenes: Catalytic Substitutions of Acetylenic Hydrogen with Bromoalkenes, Iodoarenes and Bromopyridines. Tetrahedron Letters, 16, 4467.

[13] Zhang, G.-P. and Li, P.-H. (2010) Pd(OAc) ${ }_{2}$ Catalyzed Sonogashira Cross-Coupling Reaction under Ultrasound Irradiation. Chinese Journal of Organic Chemistry, 30, 117-119.

[14] Li, J.-X., Wang, Z.-Y., Xue, F.-L. and Luo, S.-H. (2011) Sonogashira Coupling Reaction of 5-Substituted 3,4-dihalo-2(5H)-furanones. Acta Chimica Sinica, 69, 1688 1696.

[15] Zhao, K.-Q., Zhou, H., Y, W.-H., Wang, B.-Q. and Hu, P. (2011) Synthesis of Novel Tricyclic Fused 2(5H)-Furanone Derivatives with 1,2,3-Triazole Structure. Acta Chimica Sinica, 69, 2835-2842.

[16] Xu, W., Sun, Y.-L., Guo, M.-H., Zhang, W.-Q. and Gao, Z.-W. (2013) Montmoril- 
lonite Supported $\mathrm{Pd} / \mathrm{Cu}$ Bimetallic Nanoparticle Catalyzed Sonogashira Coupling. Chinese Journal of Organic Chemistry, 33, 820-826. https://doi.org/10.6023/cjoc201301077

[17] Jiao, L., Lin, M., Zhuo, L.-G. and Yu, Z.-X. (2010) Rh(I)-Catalyzed $[(3+2)+1]$ Cycloaddition of 1-yne/ene-vinylcyclopropanes and CO: Homologous PausonKhand Reaction and Total Synthesis of ( \pm )- $\alpha$-agarofuran. Organic Letters, 12, 2528 2531. https://doi.org/10.1021/ol100625e

[18] Cooke, J.W.B., Bright, R., Coleman, M.J. and Jenkins, K.P. (2001) Process Research and Development of a Dihydropyrimidine Dehydrogenase Inactivator: Large-Scale Preparation of Eniluracil Using a Sonogashira Coupling. Organic Process Research \& Development, 5, 383-386. https://doi.org/10.1021/op0100100

[19] Dai, W.M., Lai, K.W., Wu, A., Hamaguchi, W., Lee, M.Y.H., Zhou, L., Ishii, A. and Nishimoto, S. (2002) DNA Cleavage Potency, Cytotoxicity, and Mechanism of Action of a Novel Class of Enediyne Prodrugs. Journal of Medicinal Chemistry, 45, 758-761. https://doi.org/10.1021/jm015588e

[20] Sonogashira, K. (2002) Development of Pd-Cu Catalyzed Cross-Coupling of Terminal Acetylenes with $\mathrm{sp}^{2}$-Carbon Halides. Journal of Organometallic Chemistry, 653, 46-49.

[21] Yin, L.-X. and Liebscher, J. (2007) Carbon-Carbon Coupling Reactions Catalyzed by Heterogeneous Palladium Catalysts. Chemical Reviews, 107, 133-173. https://doi.org/10.1021/cr0505674

[22] Bunz, U.H.F. (2000) Poly(aryleneethynylene)s: Syntheses, Properties, Structures, and Applications. Chemical Reviews, 100, 1605-1644.

https://doi.org/10.1021/cr990257j

[23] Lee, S.H., Nakamura, T. and Tsutsui, T. (2001) Synthesis and Characterization of Oligo(9,9-dihexyl-2,7-fluorene ethynylene)s: For Application as Blue Light-Emitting Diode. Organic Letters, 3, 2005-2007. https://doi.org/10.1021/ol010069r

[24] Ezugwu, G.I., Mousavi, B., Asrafa, M.A., Mehta, A., Vardhan, H. and Verpoort, F. (2016) An N-Heterocyclic Carbene Based MOF Catalyst for Sonogashira CrossCoupling Reaction. Catalysis Science \& Technology, 6, 2050-2054. https://doi.org/10.1039/C5CY01944C

[25] Sun, G.-L., Lei, M. and Hu, L.-H. (2016) A Facile and Efficient Method for the Synthesis of Alkynone by Carbonylative Sonogashira Coupling Using $\mathrm{CHCl}_{3}$ as the $\mathrm{CO}$ Source. RSC Advances, 6, 28442-28446. https://doi.org/10.1039/C6RA02424F

[26] Ikeda, A., Omote, M., Kusumoto, K., Komori, M., Tarui, A., Sato, K. and Ando, A. (2016) A Dramatic Enhancing Effect of $\mathrm{InBr}_{3}$ towards the Oxidative Sonogashira Cross-Coupling Reaction of 2-Ethynylanilines. Organic \& Biomolecular Chemistry, 14, 2127-2133. https://doi.org/10.1039/C5OB02558C

[27] Yang, Z., Li, J., Yang, T. and Zhou, C.-S. (2016) CuI Assisted Desulfurative Sonogashira Reaction of Mercapto $N$-Heterocyclic Derivatives with Alkynes. RSC Advances, 6, 65775-65778. https://doi.org/10.1039/C6RA05104A

[28] Jung, Y. and Kim, I. (2016) Chemoselective Reduction of Quinols as an Alternative to Sonogashira Coupling: Synthesis of Polysubstituted Benzofurans. Organic \& Biomolecular Chemistry, 14, 10454-10472. https://doi.org/10.1039/C6OB01941B

[29] Leboho, T.-C., Vuuren, S.F., Michaela, J.P. and Koning, C.B. (2014) The Acid-Catalyzed Synthesis of 7-Azaindoles from 3-alkynyl-2-aminopyridines and Their Antimicrobial Activity. Organic \& Biomolecular Chemistry, 12, 307-315. https://doi.org/10.1039/C3OB41798K

[30] Henry, G.D. (2004) De Novo Synthesis of Substituted Pyridines. Tetrahedron, 60, 6043-6061. 
[31] Karg, E., Rosengren, E. and Rorsman, H. (1991) Hydrogen Peroxide as a Mediator of Dopac-Induced Effects on Melanoma Cells. Journal of Investigative Dermatology, 96, 224-227. https://doi.org/10.1111/1523-1747.ep12462042

[32] Wakamatsu, K., Tabuchi, K., Ojika, M., Fabio, A.Z., Luigi, Z. and Shosuke, I. (2015) Norepinephrine and Its Metabolites Are Involved in the Synthesis of Neuromelanin Derived from the Locus Coeruleus. Journal of Neurochemistry, 135, 768-776. https://doi.org/10.1111/jnc.13237

[33] Zhao, S.-B. and Wang, S. (2010) Luminescence and Reactivity of 7-Azaindole Derivatives and Complexes. Chemical Reviews, 39, 3142-3156.

https://doi.org/10.1039/c001897j

\section{Scientific Research Publishing}

Submit or recommend next manuscript to SCIRP and we will provide best service for you:

Accepting pre-submission inquiries through Email, Facebook, LinkedIn, Twitter, etc. A wide selection of journals (inclusive of 9 subjects, more than 200 journals) Providing 24-hour high-quality service User-friendly online submission system Fair and swift peer-review system Efficient typesetting and proofreading procedure Display of the result of downloads and visits, as well as the number of cited articles Maximum dissemination of your research work

Submit your manuscript at: http://papersubmission.scirp.org/

Or contact mrc@scirp.org 